\title{
Energy Harvesting Multiple Access Channel with Data Arrivals
}

\author{
Berk Gurakan \\ Sennur Ulukus \\ Department of Electrical and Computer Engineering \\ University of Maryland, College Park, MD 20742 \\ gurakan@umd.edu \\ ulukus@umd.edu
}

\begin{abstract}
We consider the energy harvesting two user Gaussian multiple access channel (MAC), where both of the users harvest energy from nature and their data packets arrive intermittently over time. We find the optimal offline transmit power and rate allocations that maximize the sum rate. First, we show that the optimization problem can be formulated in terms of the data rates only, instead of both transmission powers and data rates. Next, we show that the optimal sum rates are non-decreasing in time, similar to the single-user optimal powers. Then, we use a dual decomposition method to solve this problem efficiently. Specifically, we show that this problem is equivalent to three subproblems where each subproblem is a throughput maximization problem with fading, data and energy arrival constraints. We decompose the problem into inner and outer optimization problems and solve the overall problem using the subgradient descent method. Finally, we consider a relaxed problem where the data and energy arrivals to both of the users are merged into single energy and data queues and show that the optimal sum rates of the original problem are majorized by the solution to this relaxed problem.
\end{abstract}

\section{INTRODUCTION}

We consider the energy harvesting multiple access channel (MAC), Fig. 1, where data packets as well as harvested energies arrive at the transmitters intermittently over time. We determine the optimum power and rate allocation policies of the users in order to maximize the sum rate of the system.

There has been a considerable amount of recent work in power control for energy harvesting communications [1]-[22]. In [1], the transmission completion time minimization problem is solved for an unlimited-sized battery. In [2], the throughput maximization problem is solved and its equivalence to the transmission completion time minimization problem is shown for an arbitrarily-sized battery. In [3], [5]-[10] the problem is extended to fading, broadcast, multiple access and interference channels. Throughput maximization problem with battery imperfections is considered in [11], [12] and processing costs are incorporated in [13]-[15]. Two-hop communication is considered with energy harvesting nodes for half- or fullduplex relay settings in [16]-[21]. Energy cooperation is introduced in [22]. Of particular relevance to us are references [8], [9], [23] where optimal scheduling problems on a MAC are investigated. In [23], minimum energy scheduling problem over a MAC where data packets arrive over time is solved. In [8], a MAC with energy arrivals is considered but it is

This work was supported by NSF Grants CNS 13-14733, CCF 14-22111 and CCF 14-22129.

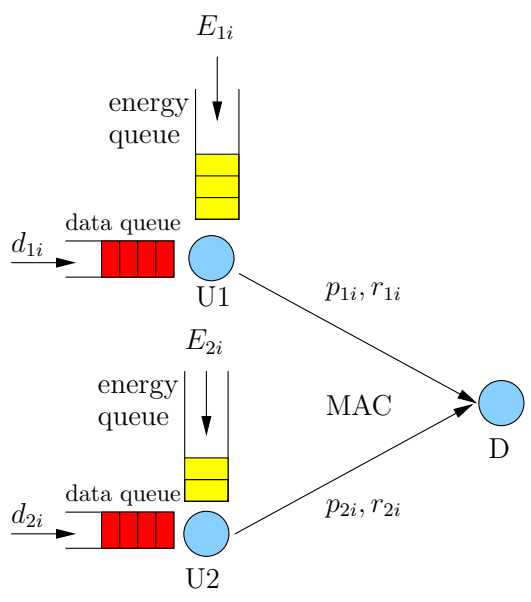

Fig. 1. Multiple access channel (MAC) with energy and data arrivals

assumed that the users are infinitely backlogged, i.e., the data packets do not arrive over time. In [9], an energy harvesting MAC with additional maximum power constraints on each user is considered. These previous works consider either data arrivals or energy arrivals but not both; in our current work, we consider both constraints.

In this paper, we first show that the optimization problem can be formulated in terms of the data rates only, instead of both transmission powers and data rates. Next, we show that the optimal sum rate is non-decreasing in time; this is similar to the single-user optimal powers [1]. Then, we use a dual decomposition method to solve this problem efficiently. Specifically, we show that this problem is equivalent to three subproblems where each subproblem is a throughput maximization problem with fading, data and energy arrival constraints. We decompose the problem into inner and outer optimization problems and solve the overall problem using the subgradient descent method.

Finally, we consider a relaxed problem where the data and energy arrivals to both of the users are merged into single energy and data queues. Without the data arrivals, it was observed in [8], that the optimal sum rates are equal to the single-user optimal rates with the energies from both users merged. This may naturally suggest that, with the presence of data causality constraints, the optimal sum rates may be given by the single-user optimal rates with both data and 
energy arrivals merged. We show that this suggestion is not entirely valid, but a majorization relationship exists between these two solutions. In particular, we show that at every slot, the optimal rates for the MAC problem transmit less total data than the single user rates with data and energy arrivals merged. Furthermore, we show that, if there exists a slot such that the optimal rates for the MAC problem and the single user relaxed problem transmit the same amount of data, then the vector of single-user rates are majorized by the optimal MAC rates.

\section{System Model and Problem Formulation}

We consider the energy harvesting MAC with intermittent data and energy arrivals as shown in Fig. 1. The harvested energies are saved in the corresponding batteries. The physical layer is a Gaussian MAC with unit-variance Gaussian noise, whose capacity region with transmitter powers $p_{1}, p_{2}$ is given by $[8]$

$$
\begin{aligned}
\mathcal{C}_{M A C}\left(p_{1}, p_{2}\right)=\left\{r_{1} \leq f\left(p_{1}\right), r_{2} \leq f\left(p_{2}\right),\right. \\
\left.r_{1}+r_{2} \leq f\left(p_{1}+p_{2}\right)\right\}
\end{aligned}
$$

where $f(x)=\frac{1}{2} \log (1+x)$. There are $N$ unit-length slots. We use subscripts 1 and 2 to denote the parameters of users 1 and 2. In slot $i$, there are energy and data arrivals to both users with amounts $E_{1 i}, E_{2 i}$ and $d_{1 i}, d_{2 i}$, respectively. We denote the transmission powers and data rates of users 1 and 2 as $p_{1 i}, r_{1 i}$ and $p_{2 i}, r_{2 i}$, respectively. We denote these power and rate sequences with the vectors $\mathbf{p}_{1}, \mathbf{p}_{2}, \mathbf{r}_{1}, \mathbf{r}_{2}$.

The energy that has not arrived yet cannot be used, leading to the following energy causality constraints:

$$
\begin{aligned}
& \sum_{i=1}^{k} p_{1 i} \leq \sum_{i=1}^{k} E_{1 i}, \quad 1 \leq k \leq N \\
& \sum_{i=1}^{k} p_{2 i} \leq \sum_{i=1}^{k} E_{2 i}, \quad 1 \leq k \leq N
\end{aligned}
$$

The data that has not arrived yet cannot be transmitted, leading to the following data causality constraints:

$$
\begin{aligned}
& \sum_{i=1}^{k} r_{1 i} \leq \sum_{i=1}^{k} d_{1 i}, \quad 1 \leq k \leq N \\
& \sum_{i=1}^{k} r_{2 i} \leq \sum_{i=1}^{k} d_{2 i}, \quad 1 \leq k \leq N
\end{aligned}
$$

The rate allocations must be achievable for the MAC in each slot:

$$
\left(r_{1 i}, r_{2 i}\right) \in \mathcal{C}_{M A C}\left(p_{1 i}, p_{2 i}\right), \quad \forall i
$$

We aim to maximize the sum rate:

$$
\begin{aligned}
\max _{\mathbf{p}_{1}, \mathbf{p}_{2}, \mathbf{r}_{1}, \mathbf{r}_{2}} & \sum_{i=1}^{N} r_{1 i}+r_{2 i} \\
\text { s.t. } & (2)-(6)
\end{aligned}
$$

\section{Formulation In TERMS OF RATES ONLY}

We start this section by reformulating the problem in terms of the rates only. We consider the following energy causality constraints on the rates:

$$
\begin{gathered}
\sum_{i=1}^{k} 2^{2 r_{1 i}}-1 \leq \sum_{i=1}^{k} E_{1 i}, \quad \forall k \\
\sum_{i=1}^{k} 2^{2 r_{2 i}}-1 \leq \sum_{i=1}^{k} E_{2 i}, \quad \forall k \\
\sum_{i=1}^{k} 2^{2\left(r_{1 i}+r_{2 i}\right)}-1 \leq \sum_{i=1}^{k} E_{1 i}+E_{2 i}, \quad \forall k
\end{gathered}
$$

and the corresponding throughput maximization problem:

$$
\begin{aligned}
\max _{r_{1 i}, r_{2 i}} & \sum_{i=1}^{N} r_{1 i}+r_{2 i} \\
\text { s.t. } & (4),(5),(8)-(10)
\end{aligned}
$$

The following lemma, proved in Appendix A, shows that this is an equivalent representation for the problem in (7).

\section{Lemma 1 The problems in (7) and (11) are equivalent.}

We solve the problem in (11) in the remainder of this paper. We denote the optimal solution to $(11)$ as $\left(r_{1 i}^{*}, r_{2 i}^{*}\right)$. We have the following lemma.

Lemma 2 The optimal sum rate is non-decreasing in time, i.e., $r_{1 i}^{*}+r_{2 i}^{*} \leq r_{1, i+1}^{*}+r_{2, i+1}^{*}, \forall i$.

Proof: The proof follows by contradiction. Assume that there is a slot $k$ such that $r_{1 k}^{*}+r_{2 k}^{*}>r_{1, k+1}^{*}+r_{2, k+1}^{*}$. We will show that this policy cannot be optimal. There can be three cases, case 1: $r_{1 k}^{*}>r_{1, k+1}^{*}, r_{2 k}^{*} \leq r_{2, k+1}^{*}$, case 2: $r_{2 k}^{*}>$ $r_{2, k+1}^{*}, r_{1 k}^{*} \leq r_{1, k+1}^{*}$ and case 3: $r_{2 k}^{*}>r_{2, k+1}^{*}, r_{1 k}^{*}>r_{1, k+1}^{*}$. Assume that the first case happens. Consider the modified policy $\hat{r}_{1 k}=\hat{r}_{1, k+1}=\frac{r_{1 k}^{*}+r_{1, k+1}^{*}}{2}$. This modified policy is feasible and transmits the same amount of data as $r_{1 i}^{*}, r_{2 i}^{*}$, but due to the convexity of the functions $2^{2\left(r_{1 i}+r_{2 i}\right)}$ and $2^{2 r_{1 i}}$, consumes less energy. This additional energy can be used to transmit more data and therefore the policy $\left(r_{1 i}^{*}, r_{2 i}^{*}\right)$ cannot be optimal. For the second case, we set $\hat{r}_{2 k}=\hat{r}_{2, k+1}=$ $\frac{r_{2 k}^{*}+r_{2, k+1}^{*}}{2}$ and for the third case we modify both $r_{1 k}, r_{1, k+1}$ and $r_{2 k}, r_{2, k+1}$ to reach a similar contradiction.

\section{ITERATIVE SOLUTION}

In this section, we will solve the problem by utilizing a dual decomposition method. The problem in (11) is equivalent to:

$$
\begin{aligned}
\max _{r_{1 i}, r_{2 i}, w_{i}} & \sum_{i=1}^{N} w_{i} \\
\text { s.t. } & (4),(5),(8),(9) \\
& \sum_{i=1}^{k} 2^{2 w_{i}}-1 \leq \sum_{i=1}^{k} E_{1 i}+E_{2 i}, \quad \forall k
\end{aligned}
$$




$$
w_{i}=r_{1 i}+r_{2 i}, \quad \forall i
$$

which can be relaxed to:

$$
\begin{aligned}
\max _{r_{1 i}, r_{2 i}, w_{i}} & \sum_{i=1}^{N} w_{i} \\
\text { s.t. } \quad & (4),(5),(8),(9) \\
& \sum_{i=1}^{k} 2^{2 w_{i}}-1 \leq \sum_{i=1}^{k} E_{1 i}+E_{2 i}, \quad \forall k \\
& w_{i} \leq r_{1 i}+r_{2 i}, \quad \forall i
\end{aligned}
$$

since at slots where the last inequality is not satisfied with equality, $r_{1 i}$ and $r_{2 i}$ can be decreased until equality is satisfied without changing the throughput. The problem in (13) is convex since the objective function is linear and the constraints are convex constraints. Denote the following sets:

$$
\begin{aligned}
& \mathcal{R}_{1}=\left\{r_{1 i} \in \mathbb{R}^{+}: r_{1 i} \text { satisfies }(4),(8)\right\} \\
& \mathcal{R}_{2}=\left\{r_{2 i} \in \mathbb{R}^{+}: r_{2 i} \text { satisfies }(5),(9)\right\} \\
& \mathcal{R}_{w}=\left\{w_{i} \in \mathbb{R}^{+}: \sum_{i=1}^{k} 2^{2 w_{i}}-1 \leq \sum_{i=1}^{k} E_{1 i}+E_{2 i}, \forall k\right\}
\end{aligned}
$$

Now, we write the partial Lagrangian function corresponding only to the last constraint in (13) as follows:

$$
\mathcal{L}\left(\mathbf{r}_{1}, \mathbf{r}_{2}, \mathbf{w}, \gamma\right)=-\sum_{i=1}^{N} w_{i}+\sum_{i=1}^{N} \gamma_{i}\left(w_{i}-r_{1 i}-r_{2 i}\right)
$$

Now, the dual function is [24]:

$$
\begin{aligned}
g(\boldsymbol{\gamma})= & \min _{r_{1 i} \in \mathcal{R}_{1}, r_{2 i} \in \mathcal{R}_{2}, w_{i} \in \mathcal{R}_{w}} \mathcal{L}\left(\mathbf{r}_{1}, \mathbf{r}_{2}, \mathbf{w}, \boldsymbol{\gamma}\right) \\
= & \min _{r_{1 i} \in \mathcal{R}_{1}}-\sum_{i=1}^{N} \gamma_{i} r_{1 i}+\min _{r_{2 i} \in \mathcal{R}_{2}}-\sum_{i=1}^{N} \gamma_{i} r_{2 i} \\
& +\min _{w_{i} \in \mathcal{R}_{w}} \sum_{i=1}^{N}\left(\gamma_{i}-1\right) w_{i} \\
= & -\max _{r_{1 i} \in \mathcal{R}_{1}} \sum_{i=1}^{N} \gamma_{i} r_{1 i}-\max _{r_{2 i} \in \mathcal{R}_{2}} \sum_{i=1}^{N} \gamma_{i} r_{2 i} \\
& -\max _{w_{i} \in \mathcal{R}_{w}} \sum_{i=1}^{N}\left(1-\gamma_{i}\right) w_{i}
\end{aligned}
$$

For fixed $\gamma$, we define the following subproblems:

$$
\begin{aligned}
& g_{1}(\gamma)=\max _{r_{1 i} \in \mathcal{R}_{1}} \sum_{i=1}^{N} \gamma_{i} r_{1 i} \\
& g_{2}(\gamma)=\max _{r_{2 i} \in \mathcal{R}_{2}} \sum_{i=1}^{N} \gamma_{i} r_{2 i} \\
& g_{3}(\gamma)=\max _{w_{i} \in \mathcal{R}_{w}} \sum_{i=1}^{N}\left(1-\gamma_{i}\right) w_{i}
\end{aligned}
$$

Now, we show that in addition to $\gamma \geq \mathbf{0}$, which follows since $\gamma$ is Lagrange multiplier, we must also have $\gamma \leq \mathbf{1}$.
Lemma 3 An optimal dual variable $\gamma^{*}$ satisfies $\gamma_{i}^{*} \leq 1, \forall i$.

Proof: Assume otherwise that $\gamma_{k}^{*}>1$ for some $k$. This implies from (23) that $w_{k}^{*}=0$. From duality $w_{k}^{*}$ also solves (13). For fixed $r_{1 i}, r_{2 i}$, the optimal $w_{i}$ in (13) can be obtained by directional waterfilling on energy levels $E_{1 i}+E_{2 i}$ with ceiling levels capped at $r_{1 i}+r_{2 i}$, which means $w_{k}^{*}=0$ is only possible if $r_{1 k}^{*}+r_{2 k}^{*}=0$. From Lemma 2, we have $r_{1 i}^{*}+r_{2 i}^{*}$ nondecreasing, thus $r_{1 k}^{*}+r_{2 k}^{*}=0$ implies $r_{1 i}^{*}+r_{2 i}^{*}=0, \forall i \leq$ $k$. This cannot be optimal if there is a non-zero energy and data arrival in the first slot, which we assume to be the case. Therefore, we have $\gamma_{k}^{*} \leq 1, \forall k$.

Slater's condition holds for the problem in (13) [24]. Therefore, there is no duality gap and the optimal values of the dual problem and the primal problem are the same. This implies that (13) is equivalent to the following problem:

$$
\max _{\mathbf{0} \leq \boldsymbol{\gamma} \leq \mathbf{1}} g(\boldsymbol{\gamma})
$$

or equivalently:

$$
\min _{\mathbf{0} \leq \boldsymbol{\gamma} \leq \mathbf{1}} h(\gamma)
$$

where $h(\gamma) \triangleq g_{1}(\gamma)+g_{2}(\gamma)+g_{3}(\gamma)$. We observe that for fixed $\gamma$ we can solve the subproblems independently. We solve the problem in (25) by separately solving the outer minimization and inner maximization problems.

\section{A. Inner Maximization}

Here we focus on the inner problems (21)-(23). We start by analyzing (21), which is:

$$
\begin{aligned}
\max _{r_{1 i}} & \sum_{i=1}^{N} \gamma_{i} r_{1 i} \\
\text { s.t. } & \sum_{i=1}^{k} r_{1 i} \leq \sum_{i=1}^{k} d_{1 i}, \quad \forall k \\
& \sum_{i=1}^{k} 2^{2 r_{1 i}}-1 \leq \sum_{i=1}^{k} E_{1 i}, \quad \forall k
\end{aligned}
$$

The problem in (26) is a single-user throughput maximization problem with data arrivals $d_{1 i}$, energy arrivals $E_{1 i}$ and generalized fading levels as $\gamma_{i}$, similar to the problem in [3]. This problem can be solved by directional waterfilling the data arrivals with base levels $\gamma_{i}^{-1}$ and then the energy arrivals with base water levels $\gamma_{i}^{-1}$ and finally taking the minimum of the two solutions. Any unused data or energy must be carried over to the future slots. The optimality of this algorithm can be shown similar to [1, Theorem 3]. The problem in (22) has the same structure and is similarly solved. The problem in (23) has only energy harvesting constraints and is solved directly using the approach in [3] with fading levels $\left(1-\gamma_{i}\right)^{-1}$ and energy levels $E_{1 i}+E_{2 i}$. Since $0 \leq \gamma_{i} \leq 1$, the base water levels are always non-negative. Denote the solutions to $g_{1}(\gamma), g_{2}(\gamma), g_{3}(\gamma)$ as $r_{1 i}^{*}(\gamma), r_{2 i}^{*}(\gamma)$ and $w_{i}^{*}(\gamma)$, respectively. Once the optimal solutions are found, we have $g_{1}(\gamma)=\sum_{i} \gamma_{i} r_{1 i}^{*}(\gamma)$, and $g_{2}(\gamma)=\sum_{i} \gamma_{i} r_{2 i}^{*}(\gamma)$, and $g_{3}(\gamma)=\sum_{i}\left(1-\gamma_{i}\right) w_{i}^{*}(\gamma)$. 


\section{B. Outer Minimiziation}

The outer minimization problem is the problem of finding optimal $\gamma$ in (25). For this problem we will use the normalized subgradient method, which is defined as

$$
\gamma^{k+1}=\gamma^{k}-\alpha_{k} \frac{\boldsymbol{q}^{k}}{\left\|\boldsymbol{q}^{k}\right\|}
$$

where $\gamma^{k+1}$ is the $k$ th iterate, $\boldsymbol{q}^{k}$ is any subgradient of $h$ at $\gamma^{k}$ and $\alpha_{k}>0$ is the $k$ th step size. For completeness, first we define the subgradient of a function: $\boldsymbol{q}$ is a subgradient of $h$ at $\boldsymbol{x}$ if [24, Eq. (6.20)]

$$
h(\boldsymbol{y}) \geq h(\boldsymbol{x})+\boldsymbol{q}^{\boldsymbol{\top}}(\boldsymbol{y}-\boldsymbol{x}), \quad \forall \boldsymbol{y}
$$

Now we show that a subgradient for $h(\gamma)$ is readily available once the inner maximization problems are solved.

Lemma 4 The vector $\mathbf{r}_{1}^{*}\left(\gamma^{k}\right)+\mathbf{r}_{2}^{*}\left(\gamma^{k}\right)-\mathbf{w}^{*}\left(\gamma^{k}\right)$ is a subgradient for $h(\gamma)$ at $\gamma^{k}$.

Proof: Similar to the discussion that follows [25, Section 6.1, Eq. (1.1)] we have:

$$
\begin{aligned}
h(\gamma) \geq & \sum_{i=1}^{N}\left[\gamma_{i} r_{1 i}^{*}\left(\gamma^{k}\right)+\gamma_{i} r_{2 i}^{*}\left(\gamma^{k}\right)+\left(1-\gamma_{i}\right) w_{i}^{*}\left(\gamma^{(k)}\right)\right] \\
= & \sum_{i=1}^{N}\left[\gamma_{i}^{k} r_{1 i}^{*}\left(\gamma^{k}\right)+\gamma_{i}^{k} r_{2 i}^{*}\left(\gamma^{k}\right)+\left(1-\gamma_{i}^{k}\right) w_{i}^{*}\left(\gamma^{(k)}\right)\right] \\
& +\sum_{i=1}^{N}\left(\gamma_{i}-\gamma_{i}^{k}\right)\left(r_{1 i}^{*}\left(\boldsymbol{\gamma}^{k}\right)+r_{2 i}^{*}\left(\boldsymbol{\gamma}^{k}\right)-w_{i}^{*}\left(\boldsymbol{\gamma}^{(k)}\right)\right) \\
= & h\left(\boldsymbol{\gamma}^{k}\right)+\left(\mathbf{r}_{1}^{*}\left(\boldsymbol{\gamma}^{k}\right)+\mathbf{r}_{2}^{*}\left(\boldsymbol{\gamma}^{k}\right)-\mathbf{w}^{*}\left(\boldsymbol{\gamma}^{k}\right)\right)^{\top}\left(\boldsymbol{\gamma}-\boldsymbol{\gamma}^{k}\right)
\end{aligned}
$$

where the inequality follows from the fact that $r_{1 i}^{*}\left(\gamma^{k}\right) \in \mathcal{R}_{1}$ so feasible for $g_{1}(\gamma)$ but may not solve $g_{1}(\gamma), r_{2 i}^{*}\left(\gamma^{k}\right) \in \mathcal{R}_{2}$ but may not solve $g_{2}(\gamma)$, and $w_{i}^{*}\left(\gamma^{(k)}\right) \in \mathcal{R}_{w}$ but may not solve $g_{3}(\gamma)$.

Using this subgradient, the method in (27) reduces to:

$$
\gamma^{k+1}=\left\{\gamma^{k}-\alpha_{k} \frac{\mathbf{r}_{1}^{*}\left(\gamma^{k}\right)+\mathbf{r}_{2}^{*}\left(\gamma^{k}\right)-\mathbf{w}^{*}\left(\boldsymbol{\gamma}^{k}\right)}{\left\|\mathbf{r}_{1}^{*}\left(\boldsymbol{\gamma}^{k}\right)+\mathbf{r}_{2}^{*}\left(\boldsymbol{\gamma}^{k}\right)-\mathbf{w}^{*}\left(\boldsymbol{\gamma}^{k}\right)\right\|}\right\}^{\oplus}
$$

where the operator $\oplus$ is defined as $x^{\oplus} \triangleq \max \{0, \min \{x, 1\}\}$ and it is the projection of $x$ to the set $0 \leq x \leq 1$. This is done so that the updates of $\gamma$ stay in the feasible range. We note that the subgradient method is not a descent method, i.e., the iterations at every step do not necessarily decrease the objective value. Therefore, it is necessary to keep track of the best point found so far. At each step, we set:

$$
h_{\text {best }}^{k}=\min \left\{h_{\text {best }}^{k-1}, h\left(\gamma^{k}\right)\right\}
$$

We denote $\gamma_{\text {best }}^{k}$ as the argument of $h_{\text {best }}^{k}$. It can be shown that for appropriately selected $\alpha_{k}, h_{\text {best }}^{k} \rightarrow h^{*}$ [25, Section 6.3]. Furthermore, if the step size $\alpha_{k}$ is chosen such that

$$
\sum_{k=1}^{\infty} \alpha_{k}=\infty
$$

$$
\sum_{k=1}^{\infty} \alpha_{k}^{2}<\infty
$$

then $\gamma_{\text {best }}^{k} \rightarrow \gamma^{*}$ [26, Proposition 5.1]. Once the optimal $\gamma^{*}$ is found, $w_{i}^{*}\left(\boldsymbol{\gamma}^{*}\right)$ is the optimal sum rate and we can find $r_{1 i}^{*}\left(\gamma^{*}\right), r_{2 i}^{*}\left(\gamma^{*}\right)$ as the optimal solutions. If $r_{1 k}^{*}\left(\gamma^{*}\right)+$ $r_{2 k}^{*}\left(\boldsymbol{\gamma}^{*}\right)>w_{k}^{*}\left(\boldsymbol{\gamma}^{*}\right)$ for some slot $k$ then we can decrease first or second user rates until equality is achieved.

\section{Relaxed Problem and Majorization}

Without the data causality constraints of (4) and (5) it was observed in [8], that the optimal sum rate is equal to the single-user optimal rate with the energies merged as $E_{1 i}+E_{2 i}$. This may naturally suggest that, with the presence of the data causality constraints, the optimal sum rate is given by the single-user optimal rate with both data and energy causality constraints. In this section, we show that this suggestion is not entirely valid, but a majorization relationship exists between these two solutions. Consider the following problem:

$$
\begin{aligned}
\max _{q_{i}} & \sum_{i=1}^{N} q_{i} \\
\text { s.t. } & \sum_{i=1}^{k} 2^{2 q_{i}}-1 \leq \sum_{i=1}^{k} E_{1 i}+E_{2 i}, \quad \forall k \\
& \sum_{i=1}^{k} q_{i} \leq \sum_{i=1}^{k} d_{1 i}+d_{2 i}, \quad \forall k
\end{aligned}
$$

This problem can be solved using the geometric approach in [1] or the directional waterfilling with both data and energy arrivals in [3]. We note that the problem in (34) is a relaxed version of (11) where the energy arrivals and data arrivals are merged to a single-user. I.e., we sum up (4) and (5) to obtain a single data arrival constraint and remove (8) and (9). We denote the solution to (34) as $q_{i}^{*}$. Now, we show a weak majorization result whose proof is provided in Appendix B.

Lemma 5 We must have $\sum_{i=1}^{k} r_{1 i}^{*}+r_{2 i}^{*} \leq \sum_{i=1}^{k} q_{i}^{*}, \forall k$.

Lemma 6 If at any slot $k$, we have $\sum_{i=1}^{k} r_{1 i}^{*}+r_{2 i}^{*}=\sum_{i=1}^{k} q_{i}^{*}$, then $\sum_{i=1}^{k} 2^{2\left(r_{1 i}^{*}+r_{2 i}^{*}\right)} \geq \sum_{i=1}^{k} 2^{2 q_{i}^{*}}$. If in addition, we have $\sum_{i=1}^{k} 2^{2 q_{i}^{*}}-1=\sum_{i=1}^{k} E_{1 i}+E_{2 i}$, then we must have $r_{1 i}^{*}+$ $r_{2 i}^{*}=q_{i}^{*}$ for $i=1, \ldots, k$.

Proof: The proof follows from majorization theory. We know that $r_{1 i}^{*}+r_{2 i}^{*}$ and $q_{i}^{*}$ are non-decreasing in $i$, so they are ordered vectors. From Lemma 5, we have $\sum_{i=1}^{l} r_{1 i}^{*}+r_{2 i}^{*} \leq \sum_{i=1}^{l} q_{i}^{*}$ $\forall l<k$ and if in addition we have $\sum_{i=1}^{k} r_{1 i}^{*}+r_{2 i}^{*}=\sum_{i=1}^{k} q_{i}^{*}$ this means that the vector $q_{i}^{*}$ is majorized by the vector $r_{1 i}^{*}+r_{2 i}^{*}$. This means $\sum_{i=1}^{k} g\left(r_{1 i}^{*}+r_{2 i}^{*}\right) \geq \sum_{i=1}^{k} g\left(q_{i}^{*}\right)$ for any convex, increasing $g$ and this is true for $g=2^{x}$ [27, Section I.3.C1B]. Furthermore, if we have $\sum_{i=1}^{k} 2^{2 q_{i}^{*}}-1=$ $\sum_{i=1}^{k} E_{1 i}+E_{2 i}$, then we have $\sum_{i=1}^{k} 2^{2\left(r_{1 i}^{*}+r_{2 i}^{*}\right)}-1 \geq$ $\sum_{i=1}^{k} E_{1 i}+E_{2 i}$. From energy feasibility of $r_{1 i}^{*}+r_{2 i}^{*}$ we also have $\sum_{i=1}^{k} 2^{2\left(r_{1 i}^{*}+r_{2 i}^{*}\right)}-1 \leq \sum_{i=1}^{k} E_{1 i}+E_{2 i}$. These two 


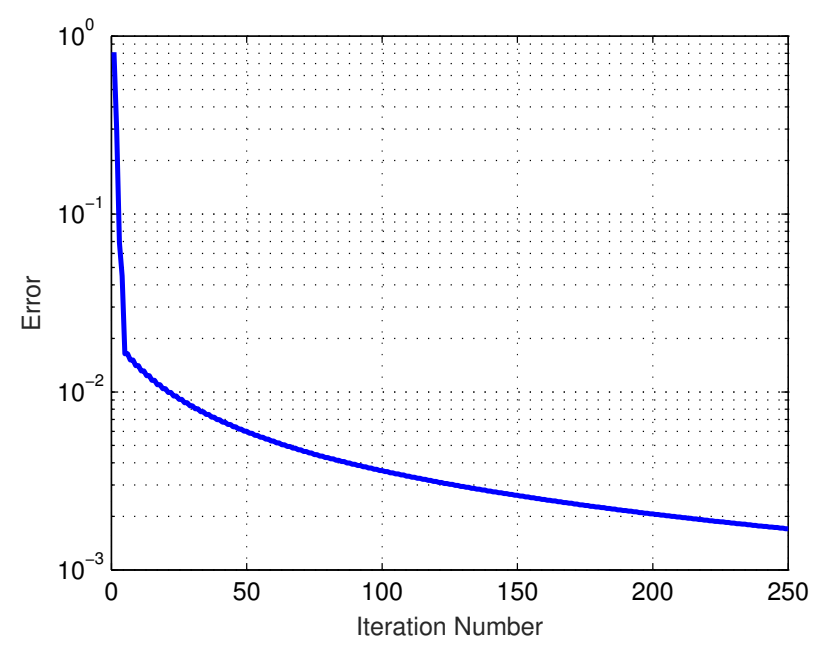

Fig. 2. Error between the best iteration so far and the optimal value

constraints are feasible if and only if $\sum_{i=1}^{k} 2^{2\left(r_{1 i}^{*}+r_{2 i}^{*}\right)}-1=$ $\sum_{i=1}^{k} E_{1 i}+E_{2 i}=\sum_{i=1}^{k} 2^{2 q_{i}^{*}}-1$. From the strict convexity of $2^{x}$ and therefore strict Schur-convexity of $\sum 2^{x}$ we must have $r_{1 i}^{*}+r_{2 i}^{*}=q_{i}^{*}, \forall i \leq k$.

In some special instances of the problem, Lemmas 5 and 6 can be utilized, by enforcing the constraint $r_{1 i}+r_{2 i}=q_{i}^{*}, \forall i$, replacing $r_{2 i}=q_{i}^{*}-r_{1 i}$ and solving a single-user problem.

\section{Numerical Results}

In this section we provide a numerical example over three slots and illustrate the resulting optimal policies. Let $\mathbf{E}_{1}=$ $[2,5,5], \mathbf{E}_{2}=[10,3,1], \mathbf{d}_{1}=[2.6,1.5,2], \mathbf{d}_{2}=[0.5,3.25,1]$. The evolution of our iterative algorithm is shown in Fig. 2. The step size is taken as $\alpha_{k}=\frac{0.1}{k}$. The plot shows the gap between the best iteration so far and the optimal value of the problem (13), denoted by $p^{*}$. The algorithm converges after around 50 steps to reasonable accuracy. The optimal rates are then found as $w_{i}^{*}=[1.29,1.76,1.76], r_{1 i}^{*}=[0.79,0.86,1.01], r_{2 i}^{*}=$ $[0.5,1.07,1]$. We see that $w_{i}^{*} \leq r_{1 i}^{*}+r_{2 i}^{*}, \forall i$ and at slots 2 and 3 we have $w_{i}^{*}<r_{1 i}^{*}+r_{2 i}^{*}$. Therefore, we can decrease the rates at slots 2 and 3 to achieve equality, i.e., we set $r_{1 i}^{*}=[0.79,0.69,0.76]$ and this $r_{1 i}^{*}, r_{2 i}^{*}$ is the optimal solution.

\section{CONCLUSIONS}

We considered the multiple access energy harvesting channel with intermittent data and energy arrivals. We first showed that the optimization problem can be formulated in terms of data rates only, instead of both transmission powers and data rates. Next, we showed that the optimal sum rate is nondecreasing in time. Then, we proposed a dual decomposition method to solve this problem efficiently. Specifically, we showed that this problem is equivalent to three subproblems where each subproblem is a throughput maximization problem with fading, data and energy arrival constraints. We decomposed the problem into inner and outer optimization problems and solved it using the subgradient descent method. Finally, we considered a relaxed problem where the data and energy arrivals to both users are merged into single energy and data queues. In this case, we showed that at every slot, the optimal sum rates of the original problem transmit less data than the single-user rates of this relaxed problem. Furthemore, if there exists a slot such that the optimal sum rates of the original problem and the single-user rates of the relaxed problem transmit the same amount of total data, then the single-user rates are majorized by the optimal MAC rates.

\section{APPENDIX A \\ PROOF OF LEMMA 1}

Denote the feasible set and the optimal value of the problem in (7) as $\left(\mathcal{F}_{1}, T_{1}\right)$ and that of the problem in (11) as $\left(\mathcal{F}_{2}, T_{2}\right)$. First, we show $T_{1} \leq T_{2}$. For any $\left(p_{1 i}, p_{2 i}, r_{1 i}, r_{2 i}\right) \in \mathcal{F}_{1}$, from (6) we have

$$
\begin{aligned}
& p_{1 i} \geq 2^{2 r_{1 i}}-1, \quad p_{2 i} \geq 2^{2 r_{2 i}}-1 \\
& p_{1 i}+p_{2 i} \geq 2^{2\left(r_{1 i}+r_{2 i}\right)}-1
\end{aligned}
$$

These constraints imply

$$
\begin{aligned}
\sum_{i=1}^{k} p_{1 i} & \geq \sum_{i=1}^{k} 2^{2 r_{1 i}}-1, \quad \forall k \\
\sum_{i=1}^{k} p_{2 i} & \geq \sum_{i=1}^{k} 2^{2 r_{2 i}}-1, \quad \forall k \\
\sum_{i=1}^{k} p_{1 i}+p_{2 i} & \geq \sum_{i=1}^{k} 2^{2\left(r_{1 i}+r_{2 i}\right)}-1, \quad \forall k
\end{aligned}
$$

Together with (2) and (3), (37)-(39) imply

$$
\begin{gathered}
\sum_{i=1}^{k} 2^{2 r_{1 i}}-1 \leq \sum_{i=1}^{k} E_{1 i}, \quad \forall k \\
\sum_{i=1}^{k} 2^{2 r_{2 i}}-1 \leq \sum_{i=1}^{k} E_{2 i}, \quad \forall k \\
\sum_{i=1}^{k} 2^{2\left(r_{1 i}+r_{2 i}\right)}-1 \leq \sum_{i=1}^{k} E_{1 i}+E_{2 i}, \quad \forall k
\end{gathered}
$$

This means $\left(r_{1 i}, r_{2 i}\right) \in \mathcal{F}_{2}$ and therefore $T_{1} \leq T_{2}$.

Now, we show $T_{2} \leq T_{1}$. For any $\left(r_{1 i}, r_{2 i}\right) \in \mathcal{F}_{2}$ we will find $p_{1 i}, p_{2 i}$ such that $\left(p_{1 i}, p_{2 i}, r_{1 i}, r_{2 i}\right) \in \mathcal{F}_{1}$. To accomplish this, we solve the following feasibility problem:

$$
\begin{array}{rl}
\max _{p_{1 i}, p_{2 i}} & 1 \\
\text { s.t. } & p_{1 i} \geq 2^{2 r_{1 i}}-1, \quad \forall i \\
& p_{2 i} \geq 2^{2 r_{2 i}}-1, \quad \forall i \\
& p_{1 i}+p_{2 i} \geq 2^{2\left(r_{1 i}+r_{2 i}\right)}-1, \quad \forall i \\
& \sum_{i=1}^{k} p_{1 i} \leq \sum_{i=1}^{k} E_{1 i}, \sum_{i=1}^{k} p_{2 i} \leq \sum_{i=1}^{k} E_{2 i}, \quad \forall k
\end{array}
$$

We can let $p_{1 i}+p_{2 i}=2^{2\left(r_{1 i}+r_{2 i}\right)}-1, \forall i$ without changing the optimal value of the feasibility problem. Now, we have the following set of inequalities to be satisfied:

$$
p_{1 i} \geq 2^{2 r_{1 i}}-1, \quad \forall i
$$




$$
\begin{aligned}
& p_{1 i} \leq 2^{2\left(r_{1 i}+r_{2 i}\right)}-2^{2 r_{2 i}}, \quad \forall i \\
& \sum_{i=1}^{k} p_{1 i} \leq \sum_{i=1}^{k} E_{1 i}, \quad \forall k \\
& \sum_{i=1}^{k} p_{1 i} \geq \sum_{i=1}^{k} 2^{2\left(r_{1 i}+r_{2 i}\right)}-1-E_{2 i}, \quad \forall k
\end{aligned}
$$

Now, we note that this set of inequalities is consistent by showing every lower bound is no larger than every upper bound. (44) is consistent with (45) since $2^{2(x+y)}-2^{2 y} \geq$ $2^{2 x}-1, \forall x, y \geq 0$. (44) is consistent with (46) since $r_{1 i}$ satisfies (40). (45) is consistent with (47) since $r_{2 i}$ satisfies (41) and finally (46) is consistent with (47) since $r_{1 i}, r_{2 i}$ satisfy (42). We also have $p_{1 i} \geq 0$ which is consistent with both (45) and (46) since these lower bounds are nonnegative. This feasibility problem then has a solution and there exists $p_{1 i}, p_{2 i}$ that solve (43). This means that there exists $\left(p_{1 i}, p_{2 i}, r_{1 i}, r_{2 i}\right) \in \mathcal{F}_{1}$ and $T_{2} \leq T_{1}$, proving the lemma.

\section{APPENDIX B \\ PROOF OF LEMMA 5}

The statement is true for $k=N$ because the optimal value of problem (34) is at least as large as that of (11) since any profile that is feasible for (11) is also feasible for (34). We will show that if the statement holds for slot $k$, i.e., $\sum_{i=1}^{k} r_{1 i}^{*}+$ $r_{2 i}^{*} \leq \sum_{i=1}^{k} q_{i}^{*}$ then it also holds for slot $k-1$. By induction this will imply that it is true for all $k$. Assume on the contrary that $\sum_{i=1}^{k-1} r_{1 i}^{*}+r_{2 i}^{*}>\sum_{i=1}^{k-1} q_{i}^{*}$. Together with $\sum_{i=1}^{k} r_{1 i}^{*}+$ $r_{2 i}^{*} \leq \sum_{i=1}^{k} q_{i}^{*}$ this implies $r_{1 k}^{*}+r_{2 k}^{*}<q_{k}^{*}$.

Now, we claim that we must have $\sum_{i=1}^{k-1} 2^{2\left(r_{1 i}^{*}+r_{2 i}^{*}\right)}>$ $\sum_{i=1}^{k-1} 2^{2 q_{i}^{*}}$. This is true because otherwise, up to slot $k-1$, the profile $r_{1 i}^{*}+r_{2 i}^{*}$ sends more data than $q_{i}^{*}$ and in view of the energy constraints in (34) leads to a more relaxed feasible set. This means that, the profile $q_{i}^{*}$ can be replaced with $r_{1 i}^{*}+r_{2 i}^{*}$ for slots 1 to $k-1$ and for the remaining slots $k, \ldots, N$ more data can be transmitted because there is more energy left. This contradicts the optimality of $q_{i}^{*}$, therefore we must have $\sum_{i=1}^{k-1} 2^{2\left(r_{1 i}^{*}+r_{2 i}^{*}\right)} \leq \sum_{i=1}^{k-1} 2^{2 q_{i}^{*}}$.

Note that this also means $\sum_{i=1}^{k-1} 2^{2 q_{i}^{*}}-1<\sum_{i=1}^{k-1} E_{1 i}+$ $E_{2 i}$ because otherwise $r_{1 i}^{*}+r_{2 i}^{*}$ cannot be energy feasible. From the assumption we have $\sum_{i=1}^{k-1} r_{1 i}^{*}+r_{2 i}^{*}>\sum_{i=1}^{k-1} q_{i}^{*}$, which implies $\sum_{i=1}^{k-1} q_{i}^{*}<\sum_{i=1}^{k-1} d_{1 i}+d_{2 i}$ because otherwise $r_{1 i}^{*}+r_{2 i}^{*}$ cannot be data feasible. These collectively mean that slot $k-1$ cannot be an energy or data exhausting slot for $q_{i}^{*}$ and therefore $q_{k-1}^{*}=q_{k}^{*}$. From this fact and $r_{1 i}^{*}+r_{2 i}^{*}$ is non-decreasing we have $r_{1, k-1}^{*}+r_{2, k-1}^{*} \leq r_{1 k}^{*}+r_{2 k}^{*}<q_{k}^{*}=$ $q_{k-1}^{*}$ which implies $r_{1, k-1}^{*}+r_{2, k-1}^{*}<q_{k-1}^{*}$. Together with $\sum_{i=1}^{k-1} r_{1 i}^{*}+r_{2 i}^{*}>\sum_{i=1}^{k-1} q_{i}^{*}$ this implies $\sum_{i=1}^{k-2} r_{1 i}^{*}+r_{2 i}^{*}>$ $\sum_{i=1}^{k-2} q_{i}^{*}$. Following the same reasoning as before, we have that $k-2$ is a non energy and data exhausting slot for $q_{i}^{*}$ and therefore $q_{k-2}^{*}=q_{k-1}^{*}$. We apply the same argument to reach the conclusion that $q_{1}^{*}=q_{2}^{*}=\cdots=q_{k}^{*}$ and $r_{1 i}^{*}+r_{2 i}^{*}<$ $q_{i}^{*}, \forall i \leq k$. This contradicts the assumption $\sum_{i=1}^{k-1} r_{1 i}^{*}+r_{2 i}^{*}>$ $\sum_{i=1}^{k-1} q_{i}^{*}$.

\section{REFERENCES}

[1] J. Yang and S. Ulukus, "Optimal packet scheduling in an energy harvesting communication system," IEEE Trans. Comm., vol. 60, pp. 220-230, Jan. 2012.

[2] K. Tutuncuoglu and A. Yener, "Optimum transmission policies for battery limited energy harvesting nodes," IEEE Trans. Wireless Comm., vol. 11, pp. 1180-1189, Mar. 2012.

[3] O. Ozel, K. Tutuncuoglu, J. Yang, S. Ulukus, and A. Yener, "Transmission with energy harvesting nodes in fading wireless channels: Optimal policies," IEEE JSAC, vol. 29, pp. 1732-1743, Sep. 2011.

[4] C. K. Ho and R. Zhang, "Optimal energy allocation for wireless communications with energy harvesting constraints," IEEE Trans. Signal Proc., vol. 60, pp. 4808-4818, Sep. 2012.

[5] J. Yang, O. Ozel, and S. Ulukus, "Broadcasting with an energy harvesting rechargeable transmitter," IEEE Trans. Wireless Comm., vol. 11, pp. 571-583, Feb. 2012.

[6] M. A. Antepli, E. Uysal-Biyikoglu, and H. Erkal, "Optimal packet scheduling on an energy harvesting broadcast link," IEEE JSAC, vol. 29, pp. 1721-1731, Sep. 2011.

[7] O. Ozel, J. Yang, and S. Ulukus, "Optimal broadcast scheduling for an energy harvesting rechargeable transmitter with a finite capacity battery," IEEE Trans. Wireless Comm., vol. 11, pp. 2193-2203, Jun. 2012.

[8] J. Yang and S. Ulukus, "Optimal packet scheduling in a multiple access channel with energy harvesting transmitters," Journal of Comm. and Netw., vol. 14, pp. 140-150, Apr. 2012.

[9] Z. Wang, V. Aggarwal, and X. Wang, "Iterative dynamic water-filling for fading multiple-access channels with energy harvesting," IEEE JSAC, vol. 33, pp. 382-395, Mar. 2015

[10] K. Tutuncuoglu and A. Yener, "Sum-rate optimal power policies for energy harvesting transmitters in an interference channel," Journal of Comm. and Netw., vol. 14, pp. 151-161, Apr. 2012.

[11] B. Devillers and D. Gunduz, "A general framework for the optimization of energy harvesting communication systems with battery imperfections," Journal of Comm. and Netw., vol. 14, pp. 130-139, Apr. 2012.

[12] K. Tutuncuoglu, A. Yener, and S. Ulukus, "Optimum policies for an energy harvesting transmitter under energy storage losses," IEEE JSAC, vol. 33, pp. 467-481, Mar. 2015.

[13] O. Orhan, D. Gunduz, and E. Erkip, "Energy harvesting broadband communication systems with processing energy cost," IEEE Trans. Wireless Comm., vol. 13, pp. 6095-6107, Nov. 2014.

[14] J. Xu and R. Zhang, "Throughput optimal policies for energy harvesting wireless transmitters with non-ideal circuit power," IEEE JSAC, vol. 32, pp. 322-332, Feb. 2014.

[15] O. Ozel, K. Shahzad, and S. Ulukus, "Optimal energy allocation for energy harvesting transmitters with hybrid energy storage and processing cost," IEEE Trans. Signal Proc., vol. 62, pp. 3232-3245, Jun. 2014.

[16] C. Huang, R. Zhang, and S. Cui, "Throughput maximization for the Gaussian relay channel with energy harvesting constraints," IEEE JSAC, vol. 31, pp. 1469-1479, Aug. 2013.

[17] D. Gunduz and B. Devillers, "Two-hop communication with energy harvesting," in IEEE CAMSAP, Dec. 2011.

[18] Y. Luo, J. Zhang, and K. B. Letaief, "Optimal scheduling and power allocation for two-hop energy harvesting communication systems," IEEE Trans. Wireless Comm., vol. 12, pp. 4729-4741, Sep. 2013.

[19] O. Orhan and E. Erkip, "Throughput maximization for energy harvesting two-hop networks," in IEEE ISIT, Jul. 2013.

[20] B. Varan and A. Yener, "Energy harvesting two-way communications with limited energy and data storage," in Asilomar Conf., Nov. 2014.

[21] B. Gurakan and S. Ulukus, "Energy harvesting cooperative diamond channel," in IEEE ISIT, Jun. 2015.

[22] B. Gurakan, O. Ozel, J. Yang, and S. Ulukus, "Energy cooperation in energy harvesting communications," IEEE Trans. Comm., vol. 61, pp. 4884-4898, Dec. 2013.

[23] E. Uysal-Biyikoglu and A. E. Gamal, "On adaptive transmission for energy efficiency in wireless data networks," IEEE Trans. Info. Theory, vol. 50, pp. 3081-3094, Dec. 2004.

[24] S. Boyd and L. Vandenberghe, Convex Optimization. Cambridge, 2004.

[25] D. Bertsekas, Nonlinear Programming. Athena Scientific, 1995.

[26] R. Correa and C. Lemaréchal, "Convergence of some algorithms for convex minimization," Mathematical Programming, vol. 62, no. 1-3, pp. 261-275, 1993.

[27] A. W. Marshall, I. Olkin, and B. C. Arnold, Inequalities: Theory of Majorization and Its Applications. Springer, 2011. 